\title{
COMMUNICATION
}

\section{Atteinte buccale au cours des pathologies bulleuses acquises : Critères d'urgence diagnostique et thérapeutique}

\section{Rochefort $\mathbf{J}^{1}$, Hervé $\mathbf{G}^{2}$, Agbo Godeau $\mathbf{S}^{3}$}

1 - Service d’Odontologie du Pr Descroix, Groupe Hospitalier Pitié Salpêtrière-Charles Foix, UFR Odontologie Université Paris Diderot

2 - Service d'Anatomie et de Cytologie pathologique du Pr Capron, Groupe Hospitalier Pitié Salpêtrière- Charles Foix, Université Pierre et Marie Curie

3 - Service de Stomatologie et Chirurgie Maxillo-Faciale du Pr Goudot, Groupe Hospitalier Pitié Salpêtrière-Charles Foix, Université Pierre et Marie Curie

\section{Introduction}

L'atteinte buccale, aiguë ou chronique, des maladies bulleuses, est souvent inauguratrice et parfois longtemps isolée. Elle se manifeste rarement par des bulles muqueuses intactes mais le plus souvent par des érosions post bulleuses douloureuses. Dans le cas de lésions acquises plusieurs diagnostics sont à évoquer : l'érythème polymorphe, l'érythème pigmenté fixe, les toxidermies (syndrome de Steven Johnson ou syndrome de Lyell) mais aussi les dermatoses auto immunes comme le pemphigus, la pemphigoïde cicatricielle, la dermatose à lg $A$ linéaire, l'épidermolyse bulleuse acquise ou encore le lichen plan ou le lupus bulleux [1] our laboratory in Buffalo, New York, received 239 consecutive archival cases of gingival biopsy with a clinical diagnosis of DG. These specimens were submitted to establish or rule out a diagnosis of a direct IF-positive VBD. The demographic, clinical, and microscopic findings were tabulated using established inclusion and diagnostic criteria.InRESULTS: Approximately half the number (48.1\%). Les conséquences de ces pathologies sont diverses et certaines d'entre elles, comme le syndrome de Lyell, peuvent rapidement engager le pronostic vital. II est donc nécessaire d'en faire le diagnostic et d'identifier certains critères de gravité.

\section{Observation}

Par l'illustration de plusieurs cas cliniques, nous déterminerons les étapes nécessaires au diagnostic des pathologies bulleuses pouvant se manifester au niveau de la cavité orale. Cet outil aura pour but d'aider le praticien dans la prise en charge de ces pathologies. Les 8 patients présentés avaient entre 25 et 60 ans et s'étaient tous présentés en urgence pour des douleurs buccales diffuses insomniantes associées à une altération de l'état général. L'examen clinique révélait des lésions bulleuses ainsi que des érosions post bulleuses multiples persistantes depuis plusieurs jours. Quatre de ces patients présentaient également une atteinte cutanée et deux une atteinte ophtalmique. Des examens complémentaires ont été réalisés permettant le diagnostic de trois érythèmes polymorphes, deux de pemphigoide cicatricielle, un pemphigus, un syndrome de Steven Johnson et un lupus bulleux. 


\section{Discussion}

Le délai d'apparition et la rapidité d'évolution de l'atteinte buccale sont des critères permettant d'apprécier le degré de l'urgence. L'anamnèse peut d'emblée orienter le diagnostic : une prise médicamenteuse peut suggérer une toxidermie et un antécédent herpétique récent un érythème polymorphe. Si une nécrolyse épidermique est suspectée, il est indispensable d'adresser le patient vers une structure spécialisée dans les plus brefs délais. Une fois ce diagnostic écarté, il est nécessaire d'évaluer rapidement le retentissement local et général de la maladie. En effet, une atteinte conjonctivale dans certains érythèmes polymorphes ou dans la pemphigoïde cicatricielle peut engager le pronostic visuel et nécessiter un traitement précoce. Lorsque l'atteinte est uniquement orale, l'urgence thérapeutique relative concerne surtout l'aspect symptomatique afin de soulager les douleurs, d'éviter une dénutrition et une déshydratation, pouvant nécessiter dans certains cas une hospitalisation.

Si l'orientation diagnostique peut se faire sur l'anamnèse et l'examen clinique, il est souvent indispensable de confirmer le diagnostic en biopsiant les lésions bulleuses pour effectuer une analyse anatomopathologique, une immunofluorescence directe recherchant la présence d'anticorps afin d'établir un diagnostic de maladie auto immune [2] et une recherche d'anticorps spécifiques circulants par immunofluorescence indirecte dans un prélèvement sanguin [4].

\section{Conclusion}

Le diagnostic de ces stomatites bulleuses ne doit pas être méconnu, car il est peut être porté dans le cadre d'une consultation spécialisée de pathologie orale. Le bilan initial doit permettre de confirmer le diagnostic, d'évaluer la sévérité de l'atteinte ainsi que son degré d'urgence thérapeutique.

\section{Références}

[1] L. Suresh and M. E. Neiders, "Definitive and differential diagnosis of desquamative gingivitis through direct immunofluorescence studies," J. Periodontol., vol. 83, no. 10, pp. 1270-1278, Oct. 2012.

[2] L. Vaillant and B. Hüttenberger, "Maladies bulleuses acquises de la muqueuse buccale," Feb. 2008.

[3] S. Agbo-Godeau, P. D. L. Soares, and H. Szpirglas, "Pemphigoïde cicatricielle : prise en charge en stomatologie," Feb. 2008.

[4] S. Duvert-Lehembre and P. Joly, "Les maladies bulleuses auto-immunes," Rev. Médecine Interne, vol. 35, no. 3, pp. 166-173, Mar. 2014. 